\title{
Extensão universitária: hanseníase na escola, em busca de um diagnóstico precoce
}

\author{
Maria Ambrosina Cardoso Maia ${ }^{1}$, Bruna Adriane Andrade Silva ${ }^{2}$, Rafael Chagas Silva ${ }^{3}$
}

Resumo: O projeto de extensão teve como objetivo desenvolver atividades educativas sobre hanseníase, visando o esclarecimento sobre sinais da doença e possível detecção de casos novos, de forma a avaliar a situação da hanseníase na população do ensino fundamental do município de Passos-MG. Foram selecionadas intencionalmente 10 escolas públicas com ensino fundamental da área urbana do município de Passos-MG no ano de 2018. Inicialmente realizouse palestras aos discentes e docentes para inteirá-los a respeito da hanseníase e de seu tratamento. Em um segundo momento, por meio de uma triagem, selecionou-se os alunos que possuíam lesões de pele pigmentadas pelo corpo. Esses alunos, após autorização dos pais, foram convidados a realizar o exame dermatoneurológico e, aqueles com análise sugestiva para hanseníase, encaminhados à Estratégia de Saúde da Família. Participaram das palestras 329 docentes e 2.962 discentes, sendo que 305 discentes foram submetidos ao exame dermatoneurológico. Desses, seis casos foram sugestivos para hanseníase e encaminhados para a Estratégia de Saúde da Família. Notou-se grande desconhecimento por parte da população a respeito da doença, principalmente em relação à cura. A busca ativa de casos é um método importante para o controle da hanseníase.

Palavras-chave: Hanseníase; Doença Infectocontagiosa; Relações Comunidade-Instituição

\section{Health students' perceptions on interdisciplinary work: experience at the outreach project smile on duty}

Abstract: The objective of the extension project was to develop educational activities about leprosy, aiming at the clarification of the disease symptoms and possible detection of new cases, to assess the leprosy scenario among elementary school students in the city of Passos, Minas Gerais state. Ten public elementary schools in the urban area of Passos were selected intentionally in 2018. Initially, lectures were held to students and teachers in order to brief them about leprosy and its treatment. At a second stage, students who had pigmented skin lesions on their bodies were selected through a screening. These students, after parental consent, were invited to undergo an exam dermato-neurological, and those with a suggestive analysis for leprosy were referred to the Family Health Strategy program. A total of 329 teachers and 2,962 students attended the lectures, and of these students, 305 underwent the exam dermatoneurological. Six cases were indicative of leprosy and committed to the Strategy of Family Health program. There was a significant lack of knowledge on the part of the population about the disease, mainly concerning the cure. Thus, active searching for cases is an essential method for leprosy control. demands, promoting their potential to work collectively and answer to the current demands of interdisciplinary healthcare.

Keywords: Leprosy; Communicable Diseases; Community-Institutional Relations
Originais recebidos em

10 de março de 2019

Aceito para publicação em

04 de março de 2020

1

Docente do curso de Medicina na Universidade do Estado de Minas Gerais.

2

Acadêmica do curso de Medicina na Universidade do Estado de Minas Gerais. brunaadrianeas@gmail.com (autora para correspondência)

3 Acadêmico do curso de Medicina na Universidade do Estado de Minas Gerais. 


\section{Introdução}

A hanseníase, doença infectocontagiosa causada pela bactéria Mycobacterium leprae (bacilo de Hansen), se destaca dentre as doenças causadas por agentes infecciosos ou parasitas, ditas doenças negligenciadas. Tais doenças, além de prevalecerem em condições de pobreza, contribuem para a manutenção do quadro de desigualdade, já que representam forte entrave ao desenvolvimento dos países (Ferreira, 2014; Neta et al., 2017). Antigamente denominada lepra, tal enfermidade atinge o sistema nervoso periférico (SP), de modo a causar alterações sensitivas e tegumentares as quais podem gerar incapacidades físicas e evoluir para deformidades definitivas (Neta et al., 2017).

A transmissão da hanseníase ocorre por meio de um indivíduo infectado, sem tratamento, que pelas vias áreas superiores, transmite o bacilo a outras pessoas. Estima-se que somente uma parcela da população que entra em contato com a bactéria manifesta a doença (Nunes et al., 2011). Além disso, tal enfermidade é historicamente antiga e, desde tempos remotos, era caracterizada como uma doença contagiosa e incurável com grande conotação religiosa, o que contribuiu ao longo dos séculos para estimular ainda mais a rejeição, discriminação e exclusão de indivíduos doentes da sociedade. Dessa forma, as pessoas acometidas pela lepra foram confinadas e isoladas durante séculos, o que deu origem ao estigma da doença e ao preconceito contra o doente (Cunha, 2002; Nunes et al., 2011).

Em 2016, de acordo com a Organização Mundial da Saúde (OMS), 143 países reportaram 214.783 casos novos de hanseníase, o que representa uma taxa de detecção de 2,9 casos por 100 mil habitantes. No Brasil, no mesmo ano, foram notificados 25.218 casos novos, perfazendo uma taxa de detecção de 12,2/100 mil hab. Esses parâmetros classificam o país como de alta carga para a doença, sendo o segundo com o maior número de casos novos registrados no mundo, ficando atrás da Índia (Ministério da Saúde, 2018; Souza et al., 2018). Devido a esse resultado, a hanseníase representa um problema de saúde pública no país por sua tendência a gerar incapacidades físicas associadas a problemas sociais e econômicos (Ribeiro et al., 2018).

Além disso, segundo Pinheiro et al. (2017), estima-se uma média de dois a três milhões de indivíduos em todo o mundo vivendo com alguma sequela em decorrência da hanseníase, bem como $20 \%$ de casos novos são diagnosticados com algum grau de incapacidade física, e outros $15 \%$ a desenvolverão durante ou após o tratamento com a poliquimioterapia específica para hanseníase. Nesse aspecto, o controle da hanseníase e, consequentemente, a diminuição das incapacidades e eliminação de fontes de infecção, passa pelo seu diagnóstico e tratamento precoce. Para isso, a estratégia de busca ativa de casos na coletividade é fundamental para o seu enfrentamento. De acordo com Freitas et al. (2017), os indicadores epidemiológicos, como o coeficiente de incidência de hanseníase em menores de 15 anos, a proporção de cura de casos novos e a proporção de contatos intradomiciliares de casos novos examinados, permitem acompanhar o alcance da eliminação da doença como problema de saúde pública.

Embora atualmente tenha tratamento e cura, o pouco conhecimento da população, o diagnóstico tardio e a pouca estruturação da rede de atenção em prol da integralidade da assistência em saúde voltada para a hanseníase contribuem com o quantitativo de indivíduos vivendo com sequelas.

O diagnóstico da hanseníase é essencialmente clínico e epidemiológico, sendo realizado por meio da anamnese e do exame dermatoneurológico para identificar lesões ou áreas de pele com alteração de sensibilidade e/ou comprometimento de nervos periféricos (Ministério da Saúde, 2002; Lastória \& Abreu, 2012).

No ano de 2018 o tema da Campanha para o combate a hanseníase declarada pelo Ministério da Saúde do Brasil foi "Hanseníase. Identificou. Tratou. Curou" e, em 2017, divulgou uma estratégia de trabalho para a busca 
de casos de hanseníase em escolares ${ }^{1}$ (Ministério da Saúde, 2017). Segundo as orientações do Ministério da Saúde as atividades desta campanha abrangem, orientações aos professores e escolares, sobre a doença, e mobilização da comunidade para detectar os casos de hanseníase.

Diante deste contexto é que se faz a importância do presente estudo, pois os dados apontam para a necessidade de se trazer para o espaço acadêmico o debate sobre o controle da hanseníase, e ainda a necessidade de se realizar exames na coletividade para promover a detecção precoce e, consequentemente, encaminhar para tratamento.

Assim, o presente projeto de extensão teve como objetivo desenvolver atividades educativas sobre hanseníase junto a estudantes do ensino fundamental de escolas públicas do município de Passos-MG, visando a difusão dos sinais da doença e possível detecção de casos novos.

\section{Procedimentos Metodológicos}

Os dois acadêmicos bolsistas que participaram das atividades extensionistas eram estudantes do $4^{\circ}$ período de Medicina de uma universidade pública de Minas Gerais. As atividades foram desenvolvidas de abril a dezembro de 2018.

Para obter domínio teórico e prático sobre hanseníase os dois acadêmicos receberam uma capacitação técnica sobre hanseníase de 20 horas, durante a qual foram discutidos os seguintes tópicos: história, conceito, sinais e sintomas, transmissão, diagnóstico, complicações, prevenção e desmistificação de conceitos para redução do preconceito, e o tratamento. Nesta etapa foi feita uma análise epidemiológica dos indicadores de hanseníase no município de Passos. Com o objetivo de intensificar as ações de diagnóstico, os acadêmicos fizeram acompanhamento de casos de hanseníase no Ambulatório de Referência de Hanseníase do município, por duas semanas. Esta fase ocorreu de abril a maio de 2018.

As palestras sobre hanseníase realizadas tiveram como público alvo os professores e alunos do ensino fundamental das escolas trabalhadas. Em relação à faixa etária dos estudantes, sua maioria era de 6 até 14 anos de idade. Entretanto, devido à presença de alunos repetentes, existiam alguns alunos acima de 14 anos.

Os acadêmicos prepararam o material didático que consistiu em duas palestras através de projeções usando o programa Power Point, uma dirigida aos professores, e outra dirigida aos alunos. Estas palestras foram validadas pela docente coordenadora do projeto e pelo serviço de referência técnica de hanseníase do município.

As palestras para os professores tiveram o intuito de desmistificar a doença e o estigma social, além de transmitir conhecimentos básicos sobre a hanseníase. Posteriormente, foram realizadas palestras com cada turma do ensino fundamental. Nestas palestras com os alunos, foram usadas estratégias mais lúdicas, para facilitar a aprendizagem.

Os responsáveis das escolas autorizaram de maneira formal a realização de todas as atividades do projeto.

\section{Busca ativa de casos de hanseníase}

Após a realização de cada palestra, os acadêmicos convidavam os alunos para participarem do projeto. Aqueles que aceitavam, levavam para casa o Termo de Consentimento Livre Esclarecido e a Ficha de Autoimagem², para que os responsáveis pudessem responder e encaminhar para a escola. 
Na Ficha de Autoimagem, os pais ou responsáveis pelo aluno marcavam os locais do corpo onde existiam manchas, e respondiam as perguntas sobre a ocorrência das manchas e suas características, além de dados como atributos pessoais. Esta mesma ficha é comumente utilizada para a triagem de casos de hanseníase.

Tanto o Termo de Consentimento Livre Esclarecido quanto a Ficha de Autoimagem são disponibilizados online pelo Ministério da Saúde devido a Campanha Nacional de Hanseníase, Verminose, Tracoma e Esquistossomose. que anualmente é feita por este órgão.

\section{Exame dermatoneurológico}

Após o recebimento da Ficha de Autoimagem e do Termo de Consentimento Livre Esclarecido, foi realizado o exame dermatoneurológico pelos acadêmicos extensionistas, nos alunos que apresentavam algum tipo de mancha na pele. Caso o aluno examinado apresentasse indícios da doença, ele era encaminhado para a Estratégia de Saúde da Família (ESF) para que o diagnóstico fosse feito por um profissional. Esse encaminhamento foi realizado pela enfermeira responsável pelo setor de Hanseníase no município de PassosMG. As fichas eram repassadas para o Setor de Hanseníase, o qual era responsável por identificar através do endereço a qual Unidade de Saúde da Família pertencia aquele aluno, fazendo assim as referências. No município de Passos todos os casos de hanseníase são diagnosticados, tratados e acompanhados pela ESF, o setor de hanseníase que está dentro da Coordenação de Epidemiologia do Município, e é responsável por coordenar os atendimentos, fazer as notificações dos casos comprovados, acompanhar os indicadores epidemiológicos, promover as capacitações das equipes, além de ser considerado como referência secundária para atenção primária de saúde.

O exame dermatoneurológico foi o escolhido, pois é o método mais utilizado para diagnóstico de hanseníase no ambiente médico. A avaliação dermatológica visa identificar as lesões de pele próprias da hanseníase, para pesquisar a sensibilidade nas mesmas. Já a identificação das lesões neurológicas é feita através da avaliação neurológica, e é constituída pela inspeção dos olhos, nariz, mãos e pés.

\section{Resultados e Discussão}

Foram realizadas palestras ao ensino fundamental em 10 escolas públicas de Passos - MG, tendo um número total de alunos matriculados, dentre as turmas visitadas, de 3.261 discentes. Nessas palestras, foram distribuídas 2.962 Fichas de Autoimagem e Termo de Consentimento Livre Esclarecido para os estudantes, representando uma porcentagem de 90,83\% de abrangência em relação ao total de alunos matriculados. Das fichas entregues, retornaram 305, uma porcentagem de 10,3\% em relação ao total. Desse total de 305 alunos, 6 casos, após a realização da avaliação pelos acadêmicos, foram considerados sugestivos para Hanseníase e encaminhados para a Estratégia de Saúde da Família, representando 1,96\% do total de fichas que retornaram.

Escolheu-se fazer a busca ativa de casos em escolas públicas de ensino fundamental por entender que a escola é um local privilegiado para o desenvolvimento de ações de saúde, além de se ter a possibilidade de diagnosticar a hanseníase em indivíduos abaixo de 15 anos de idade, o que é atualmente uma preocupação dos serviços de saúde. $\mathrm{O}$ acometimento de crianças pressupõe a presença de adultos doentes sem diagnóstico e sem tratamento, convivendo e transmitindo a hanseníase para crianças e adolescentes.

De acordo com Souza et. al. (2018), entre 2012 e 2016 a taxa de detecção de casos novos foi igual a 4,5/100 mil habitantes na faixa etária estudada, classificando o país como endêmico em relação à hanseníase para essa faixa. 
Em 2016, a incidência de novos casos de hanseníase perfazia um número de 12,2/100 mil habitantes no Brasil, ou seja, uma incidência de aproximadamente $0,01 \%$. Sendo assim, comparando esses dados aos obtidos, os casos sugestivos de hanseníase apresentam taxas de incidência acima da média do país em 2016 (Ministério da Saúde, 2018).

Como já é de conhecimento comum, os fatores socioeconômicos e sociais têm ampla importância na disseminação da doença e, consequentemente, na sua não erradicação (Silva et al., 2019). Dos casos sugestivos, a maioria deles foi encontrado em escolas da periferia do município. Tal resultado confirma a associação da presença da doença em populações mais vulneráveis e menos favorecidas financeiramente, sendo fatores determinantes para a presença da hanseníase as condições de moradia, a renda da população afetada e a escolaridade (Silva \& Almeida, 2018; Silva et al., 2018).

Em estudo realizado entre 2001 e 2012 no estado do Tocantins, foi constatado diagnóstico tardio em crianças, que às vezes já até apresentavam deformidades visíveis devido a esse atraso na detecção da hanseníase. Isso reflete o desconhecimento da população, bem como reafirma o que foi citado anteriormente em relação às condições sociais das crianças afetadas. Além disso, retrata a precariedade do sistema de saúde em regiões geograficamente menos favorecidas (Monteiro et al., 2019).

Em relação à sintomatologia, segundo Velôso et al. (2018), além das lesões na pele, a doença pode também lesionar os nervos periféricos, tendo como prognóstico incapacidades físicas e deformidades. Dentre os casos analisados no presente estudo, os sinais e sintomas apresentados e/ou relatados durante o exame dermatoneurológico foram: áreas de pele esbranquiçadas (hipocrômicas); alterações de sensibilidade térmica, tátil e dolorosa no local da mancha; formigamento e choque em membros superiores e inferiores; dormência; diminuição e queda de pelos no local afetado; ausência de sudorese na área esbranquiçada; perda de força muscular; e espessamento de nervos fibular e ulnar.

Nesse contexto, no que diz respeito às incapacidades físicas, o diagnóstico precoce da hanseníase é primordial para que essas sejam evitadas. Sendo assim, a busca ativa da doença faz-se de extrema importância em qualquer faixa etária, para que as chances de deformidades, incapacidades físicas e transmissão sejam minimizadas (Freitas et al., 2018).

O Ministério da Saúde do Brasil indica algumas atividades a serem feitas para minimizar os índices de caso de hanseníase no país, como por exemplo o uso correto da poliquimioterapia, a investigação dos contatos mais próximos do paciente contaminado, bem como a aplicação da vacina BCG (Bacilo de Calmette-Guérin) (Silva et al., 2019; Xavier et al., 2019). Nesse aspecto, foi identificado um grande medo dos alunos em serem diagnosticados com a doença, de forma que o preconceito dificulta a realização das medidas cabíveis, além de que alguns responsáveis não autorizaram a realização do exame.

Ademais, algumas dificuldades foram identificadas durante a execução do projeto, que prejudicaram parcialmente o andamento das atividades. Deve-se destacar a não devolução das Fichas de Autoimagem. Tal situação concentrou-se naqueles estudantes que cursam do $6^{\circ}$ ao $9^{\circ}$ ano do ensino fundamental, os quais correspondem normalmente a faixa etária de 11 a 14 anos ou mais, em que grande parte dos estudantes relataram o esquecimento ou ainda o desaparecimento do documento, demonstrando o quanto as questões de saúde são negligenciadas por essa parcela. O contrário ocorreu com estudantes em menor faixa etária, de 6 a 10 anos, e que estavam do $1^{\circ}$ ao $5^{\circ}$ ano do fundamental, em que se evidenciou um número considerável de retorno de Fichas de Autoimagem, além da qualidade do preenchimento. Esta constatação não se refere necessariamente à criança, mas aos pais ou responsáveis, uma vez que existe maior presença ativa dos responsáveis nas questões escolares dos estudantes de menor faixa etária, demonstrando também a preocupação com a doença e as suas potenciais sequelas. 
Uma outra dificuldade ocorrida, que é considerada natural nas ações de controle da hanseníase, é a dificuldade da realização do exame dermatoneurológico em faixa etária menor, o que fez com que demorasse a conclusão das atividades. Quanto menor a faixa etária, mais complexa é a realização do exame dermatoneurológico, uma vez que as respostas aos testes muitas vezes são incoerentes, o que dificulta a exatidão do exame, e a interpretação dos sinais e sintomas apresentados.

Outro dado relevante coletado durante o projeto refere-se ao número de casos relatados de hanseníase em familiares dos estudantes que responderam às Fichas de Autoimagem. De 350 fichas, 31 fizeram relato de casos de hanseníase na família. A princípio este era um dado que chamou a atenção dos acadêmicos, mas percebeu-se que ainda existia falta de conhecimento na população sobre a doença, uma vez que o simples fato de algum membro familiar possuir manchas no corpo era motivo de se considerarem afetados pela doença, mesmo sem o diagnóstico por um profissional da saúde, e isso pôde ser demonstrado durante a anamnese com o aluno.

Em um estudo realizado no estado do Pará, tendo basicamente o mesmo intuito do presente artigo, com uma amostra inicial de 200 estudantes menores de 15 anos, 17 crianças foram consideradas suspeitas para o diagnóstico de hanseníase, sendo três dessas crianças beneficiadas com o tratamento da Hanseníase (Xavier et al., 2019). Tanto eese estudo quanto o presente trabalho ilustram a realidade brasileira e mostram a relevância que a doença tem, e a necessidade de se fazer a busca ativa nesta faixa etária.

Nesse contexto, a presente atividade extensionista teve como intuito a disseminação de conhecimento sobre a hanseníase, bem como seu diagnóstico precoce, já que apenas nove dos estados brasileiros têm doenças negligenciadas como a hanseníase incluídas no currículo básico de cursos de graduação na área da saúde. Além disso, geralmente as atividades educacionais promovidas pelo governo se baseiam em distribuição de folders, e não em ações efetivas como as prestadas por esse e tantos outros estudos (Feitosa et al., 2019). É importante ressaltar o quanto tais políticas públicas são necessárias em estados como o Tocantins, em que exames de contato, campanhas em massa e outros exames coletivos precisam ser realizados para diminuir a disseminação da doença (Monteiro et al., 2019).

Sendo assim, comparando-se o estudo realizado à literatura, a cidade de Passos-MG retrata a realidade do país, de modo que a educação em saúde se faz necessária no município.

\section{Considerações finais}

Conclui-se que o presente projeto alcançou o seu objetivo no que tange a realização de todas as atividades propostas, bem como o objetivo de minimizar o estigma social que tem a doença. Além disso, a busca ativa da hanseníase mostra-se de grande importância, visto que o ambiente escolar, em que há um contato frequente e constante entre os alunos, é um ambiente propício à disseminação da doença.

Sugere-se, de acordo com a análise do projeto, a realização de mais atividades que empoderem os alunos e os professores em questões relacionadas às doenças infectocontagiosas. Diante desse fato, faz-se necessário a intensificação de ações que promovam a educação em saúde, com consequente melhoria da saúde individual e coletiva.

Considera-se, portanto, que ações de educação em saúde voltadas para o público juvenil (crianças e adolescentes) necessitam ser ampliadas, visto que corroboram com a formação de indivíduos críticos, cidadãos e agentes de transformação social. Ademais, este projeto contribuiu significativamente com a interação ensinoserviço-comunidade e com a formação social, política, acadêmica e cultural da equipe extensionista, aproximando-os da sua principal ferramenta laboral, o outro. 
As atuais Diretrizes Curriculares Nacionais para os cursos de saúde preconizam que o processo de formação dos profissionais desta área tenha como foco os principais problemas de saúde da região onde o mesmo está inserido. Assim, debater e executar ações de controle da hanseníase, doença considerada negligenciada em nosso país, é uma necessidade do meio acadêmico. Infelizmente, o que se vê no Brasil são cursos de graduação na área da saúde destinando uma carga horária mínima para que se discuta a hanseníase, sendo comum seus egressos não terem a oportunidade de examinar um portador de hanseníase durante a vida acadêmica.

Acredita-se que o presente projeto oportunizou situações de aprendizagem desta temática pelos acadêmicos participantes, cumprindo assim o papel da extensão universitária, que é trazer o conhecimento para o interior da universidade e, ao mesmo tempo, divulgar este conhecimento elaborado para a comunidade.

\section{Agradecimentos}

Ao Programa Institucional de Apoio à Extensão da Universidade do Estado de Minas Gerais - PAEx/UEMG pelo apoio financeiro por meio de bolsas de estudos, que viabilizaram a execução deste projeto. Às instituições de ensino estaduais participantes, que permitiram a execução do projeto no seu âmbito escolar.

\section{Contribuição de cada autor}

A autora MACM foi responsável pela elaboração do projeto, por coordenar e orientar a equipe extensionista, e por colaborar na escrita do presente artigo, bem como revisar o texto final deste. Os autores BAAS e RCS escreveram o presente relato de experiência do projeto, foram os responsáveis por executar o projeto, desenvolver o material utilizado para as palestras, além de realizarem as palestras e os exames dermatoneurológicos nos estudantes. Todos os autores se encontram disponíveis para o esclarecimento de dúvidas.

\section{Notas}

1. A estratégia está disponível na página seis do documento recuperado em http://portalarquivos2.saude.gov.br/images/pdf/2018/abril/23/Informe-T--cnico-e-Operacional---V-CampanhaNacional-de-Hansen--ase-Verminoses-Tracoma-e-Esquistossomose.pdf

2. A Ficha de Autoimagem utilizada para o estudo está disponível no documento recuperado em www.saude.mt.gov.br/arquivo/4879

\section{Referências}

Cunha, A. Z. S. (2002). Hanseníase: Aspectos da evolução do diagnóstico, tratamento e controle. Ciência e Saúde Coletiva, 7(2), 235-242.

Feitosa, M. C. R., Stelko-Pereira, A. C., \& De Matos, K. J. N. (2019). Validação da tecnologia educacional brasileira para disseminação de conhecimento sobre a hanseníase para adolescentes. Revista Brasileira de Enfermagem, 72(5), 14011408 .

Ferreira, I. N. (2014). A hanseníase no contexto das doenças negligenciadas. In Alves, E. D., Ferreira, T. L., \& Ferreira, I. N. (eds.) Hanseníase: Avanços e Desafios. Brasília: NESPROM, p. 41-44.

Freitas, B. H. B. M., Xavier, D. R., Cortela, D. C. B., \& Ferreira, S. M. B. (2018). Hanseníase em menores de quinze anos em municípios prioritários, Mato Grosso, Brasil. Revista Brasileira de Epidemiologia 21: e180016. 
Lastória, J. C., \& Abreu, M. A. M. M. (2012). Hanseníase: Diagnóstico e tratamento. Diagnóstico \& Tratamento, 17(4), p. 173-179.

Monteiro, L. D., Mello, F. R. M., Miranda, T. P. M., \& Heukelbach, J. (2019). Hanseníase em menores de 15 anos no estado do Tocantins, Brasil, 2001-2012: Padrão epidemiológico e tendência temporal. Revista Brasileira de Epidemiologia, 22: e190047.

Ministério da Saúde. Brasil. Secretaria de Políticas de Saúde (2002). Guia para o controle da hanseníase. Brasília: Ministério da Saúde. Recuperado de http://bvsms.saude.gov.br/bvs/publicacoes/guia de hanseniase.pdf

Ministério da Saúde. Brasil. Secretaria de Vigilância em Saúde (2017). Informe Técnico e Operacional: "V Campanha Nacional de Hanseníase, Verminoses, Tracomas e Esquistossomose". Brasília: Ministério da Saúde. Recuperado de http://portalarquivos2.saude.gov.br/images/pdf/2018/abril/23/Informe-T--cnico-e-Operacional---V-Campanha-

Nacional-de-Hansen--ase-Verminoses-Tracoma-e-Esquistossomose.pdf

Ministério da Saúde. Brasil. Secretaria de Vigilância em Saúde (2018). Boletim epidemiológico: Hanseníase. Brasília: Ministério da Saúde, 49(4). Recuperado de http://portalarquivos2.saude.gov.br/images/pdf/2018/janeiro/31/2018004-Hanseniase-publicacao.pdf

Neta, O. A. G., Arruda, G. M. M. S., Carvalho, M. M. B., \& Gadelha, R. R. M. (2017). Percepção dos profissionais de saúde e gestores sobre a atenção em hanseníase na Estratégia Saúde da Família. Revista Brasileira em Promoção da Saúde, $30(2), 239-248$.

Nunes, J. M., Oliveira, E. N., \& Vieira, N. F. C. (2011) Hanseníase: Conhecimentos e mudanças na vida das pessoas acometidas. Ciência e Saúde Coletiva, 16(1), 1311-1318.

Pinheiro, M. G. C., Miranda, F. A. N., Carvalho, F. P. B., Ataide, C. A. V., \& Lira, A. L. B. C. (2017). Compreendendo a "alta em hanseníase": Uma análise de conceito. Revista Gaúcha de Enfermagem, 38(4), e63290.

Ribeiro, M. D. A., Silva, J. C. A., \& Oliveira, S. B. (2018). Estudo epidemiológico da hanseníase no Brasil: Reflexão sobre as metas de eliminação. Revista Panamericana de Salud Publica, 42, e42.

Patrício, A. C. F. A., da Silva, D. F., Santos, G. C. V., \& Brasil, M. H. F. (2019). Causas e estratégias de soluções para hanseníase em crianças: Diagrama de Ishikawa. Revista de Pesquisa: Cuidado é Fundamental, 11(3), 739-747.

Silva, J. S. R., Palmeira, I. P. P., Sá, A. M. M., Nogueira, L. M. V., \& Ferreira, A. M. R. (2018). Fatores sociodemográficos associados ao grau de incapacidade física na hanseníase. Revista Cuidarte, 9(3), 1-11.

Silva, L. C.; \& Almeida, L. Q. (2018). Os casos de hanseníase e a vulnerabilidade social no município de Natal, RN, Brasil: Análise das ocorrências e das áreas de risco à saúde pública. Hygeia, 14(28), 13-31.

Souza, C. D. F., Santos, F. G. B., Marques, C. S., Leal, T. C., Paiva, J. P., \& Araújo, E. M. C. F. (2018). Estudo espacial da hanseníase na Bahia, 2001-2012: Abordagem a partir do modelo bayesiano empírico local. Revista Epidemiologia e Serviços de Saúde, 27(4): e2017479.

Velôso, D. S., Melo, C. B., Sá, T. L. B., Santos, J. P., Nascimento, E. F., \& Costa, F. A. C. (2018) Perfil clínico epidemiológico da hanseníase: Uma revisão integrativa. Revista Eletrônica Acervo Saúde, 10(1), 1429-1437.

Xavier, M. B., Souza, H. S. L., Costa, S. M. R., Silva, M. J. F., Miranda, N. I. F., Queiroz, M. F. De A., \& Gonçalves, C. G. da C. (2019). Diagnóstico precoce de hanseníase em crianças da vila Santo Antônio do Prata, região hiperendêmica no estado do Pará. Revista Conexão UEPG, 15(1), 37-42.

Como citar este artigo:

Maia, M. A. C., Silva, B. A. A., \& Silva, R. C. Extensão universitária: Hanseníase na escola, em busca de um diagnóstico precoce. Revista Brasileira de Extensão Universitária, 11(1), 25-32. https://periodicos.uffs.edu.br/index.php $\angle$ RBEU/article/ view/10778/pdf 